\title{
Phenomenological damping of nonlinear-optical response tensors
}

\author{
D. L. Andrews \\ School of Chemical Sciences, University of East Anglia, Norwich NR4 7TJ, United Kingdom
}

\author{
S. Naguleswaran and G. E. Stedman \\ Department of Physics and Astronomy, University of Canterbury, Private Bag 4800, Christchurch, New Zealand
}

(Received 2 September 1997)

\begin{abstract}
The damping factors that occur in nonlinear-optical response tensors are commonly assigned signs that depend upon the temporal ordering of the associated photon interactions. However, time-reversal symmetry is satisfied only if all these signs are identical. For example, only then is there a formal equivalence between the amplitudes for second-harmonic generation and parametric down-conversion and only then is electro-optic rotation forbidden in fluids. This correction reflects deficiencies in certain commonly employed semiclassical approaches to nonlinear optics. [S1050-2947(98)10406-7]

PACS number(s): 42.65.-k
\end{abstract}

\section{INTRODUCTION}

The theoretical formulation of nonlinear optical processes commonly entails representing the material response in terms of parameters characterizing both its ground and various higher-energy states. When one of the excited states differs from the initial state by any amount closely similar to the energy of one or more participating photons, resonance enhancement is observed to occur. In such cases it is necessary to include damping in the description to properly account for the finite optical amplification or the detailed dispersion behavior.

Apart from a few simple cases (see, for example, Ref. [1]) there are considerable difficulties associated with the treatment of optical damping in a nonphenomenological manner. In an ensemble situation, the various damping mechanisms, such as radiative, collisional, or intramolecular vibrational redistribution damping, will often contribute simultaneously. In principle, the formalism of quantum field theory in statistical physics $[2,3]$ will give the correct form of the transition amplitudes in such complex situations, including (as described below) the sign of the damping factors. However, detailed calculation can be a formidable task [4] and commonly only a phenomenological treatment is tractable. One pragmatic alternative (for example, Ref. [5]) is to dispense with such damping and to apply the ensuing results only in frequency regions well away from resonance. Such an approach has the attraction of retaining a rigor that confers what in other quantum mechanical areas would be termed $a b$ initio status. This approach is however limited, being not well suited to the analysis of dispersion effects.

Very close to resonance or under strong pumping conditions (when electromagnetic field strengths become comparable to internal fields), standard perturbation theory is inappropriate and a two-level model (or a multilevel extension thereof) is invariably employed to describe, for example, Rabi flopping [6,7]. Here we examine the alternative case of resonances with moderate fields, i.e., the cases where a perturbative treatment is possible and where the optical response can meaningfully be cast in susceptibility terms. In this situation, losses that usually characterize bulk or en- semble response may enter a formulation based on independent molecules so as to endow excited levels with a finite linewidth (see, for example, Ref. [8]). This leads to the association of damping factors with the excited-state wave functions, entering into the energy denominators of susceptibility tensors as imaginary addenda [9]. The magnitude of each imaginary addendum carries the physically significant connotation of the lifetime of an excited state and leads to Lorentzian line shapes of appropriate and experimentally determinable width. We accept the pragmatic value of this damping concept and are concerned with a comparison of the signs in the resulting expression with those that result from a fully quantum calculation.

It is common practice to incorporate such damping by including imaginary terms in the energy denominators that emerge from the perturbation treatment. Two conventions have been used in the literature for setting the signs of these damping factors. The most common convention is to assign signs by time-ordering considerations. For example, in second-harmonic generation, signs are chosen oppositely for interactions preceding and following the emission of the harmonic photon. This approach has been founded on a semiclassical formalism featuring the optical susceptibility [1013], sometimes via an explicit appeal to causality, but again within the context of the semiclassical formalism [14]. We show, nevertheless, that this convention is inconsistent with a fully quantum development [1], in particular with timereversal symmetry alone, and that all these signs should be identical. Furthermore, this quantum calculation may be used to justify from first principles the apparently arbitrary process of including a damping factor via an explicit consideration of all possible quantum dynamical processes. This is evident in the Green's-function approach [2] where the imaginary factors are derived as the cumulative effects of an infinite sequence of virtual excitations, through the imaginary part of the self-energy.

Since damping effects are often associated with unidirectional dissipative effects, the relevance of our appeal to timereversal invariance needs to be clarified. Pure quantum mechanics, like classical mechanics, is time-reversal invariant. Transitions between states of the unperturbed Hamiltonian 
and so the damping factors associated with lifetime effects appear in both senses of propagation (within the timereversal-invariant formalism). Our discussion centers on the signs allotted to these factors at this stage. In contrast, the arrow of time may be invoked, for example, when the degrees of freedom of a subsystem (a bath or reservoir) are averaged over. This step is not a necessary part of our analysis and does not change these signs.

\section{HISTORY OF ALTERNATIVE SIGN CONVENTIONS}

There exist two approaches in the semiclassical treatment of optical damping. The first operates at the wave-function level and is exemplified by Butcher et al. [14] and Butcher and Cotter [9] (see also Ref. [15]) who fix the signs of their damping addenda by requiring that (in the evaluation of the susceptibility tensors) time integrals should converge. For the generation of optical harmonics and in the susceptibility contribution corresponding to each time ordering, these damping terms then carry opposite signs for interactions preceding and following emission of the harmonic photon. The second approach involves the inclusion of damping factors at the density matrix level [10-13] and results in susceptibilities with an identical assignment of signs as in Refs. $[14,9,15]$, but with extra terms added. (As described below, each approach to some extent represents a phenomenological and entirely semiclassical adaptation of the quantum theoretic Green's-function formalism.) We shall treat the two approaches on the same footing since it is the signs associated with the damping factors that is of particular interest in this paper. For example, in the multipolar gauge and in electric dipole coupling, the lowest-order (linear) optical susceptibility takes the form

$$
\begin{aligned}
\chi_{\alpha \beta}^{(1)}(-\omega ; \omega)= & \sum_{g} \sum_{b}\left[\frac{\left\langle g\left|\mu_{\alpha}\right| b\right\rangle\left\langle b\left|\mu_{\beta}\right| g\right\rangle}{E_{g}-E_{b}+\hbar \omega+i \Gamma_{b}}\right. \\
& \left.+\frac{\left\langle g\left|\mu_{\beta}\right| b\right\rangle\left\langle b\left|\mu_{\alpha}\right| g\right\rangle}{E_{g}-E_{b}-\hbar \omega-i \Gamma_{b}}\right]
\end{aligned}
$$

where $\mu_{\alpha}$ is a Cartesian component of the electric dipole operator, $|g\rangle$ the (possibly degenerate) local ground state of the unperturbed material Hamiltonian, $E_{g}$ its corresponding eigenvalue, and $2 \pi / \Gamma_{b}$ the finite lifetime of the excited state $|b\rangle$ [9].

Cohen-Tannoudji et al. [1], who consider radiative damping for resonant Rayleigh scattering, employ the resolvent operator method to sum appropriate diagrams to infinite order. The resulting transition amplitude is equal to the above except that the signs of the imaginary addenda are positive in both denominators:

$$
\begin{aligned}
\mathcal{A}_{\alpha \beta}^{(1)}(-\omega ; \omega)= & \sum_{g} \sum_{b}\left[\frac{\left\langle g\left|\mu_{\alpha}\right| b\right\rangle\left\langle b\left|\mu_{\beta}\right| g\right\rangle}{E_{g}-E_{b}+\hbar \omega+i \Gamma_{b}}\right. \\
& \left.+\frac{\left\langle g\left|\mu_{\beta}\right| b\right\rangle\left\langle b\left|\mu_{\alpha}\right| g\right\rangle}{E_{g}-E_{b}-\hbar \omega+i \Gamma_{b}}\right] .
\end{aligned}
$$

Hecht and Barron [16], who cite Weisskopf [17], also arrive at this expression. Equation (2) is also consistent with the (closely related) application of quantum field theoretical methods of statistical physics to electronic spectra. It is a general result of this approach that any physically observable spectrum $\rho(\omega)$ is related to the discontinuity of the appropriate Green's function $G(z)$ as the complex argument $z$ $=\omega \pm i \varepsilon$ is taken across the real axis [18]:

$$
\rho(\omega) \propto \lim _{\varepsilon \rightarrow 0^{+}}[G(\omega-i \varepsilon)-G(\omega+i \varepsilon)] .
$$

It is a straightforward exercise [2] to verify this spectral theorem in perturbation theory. The cases of one-photon absorption [19] and two-photon absorption [4] are explicitly available. (In the latter, a choice exists for the frequency argument, reflecting the different physical processes described by a given Green's function.)

We require the imaginary parts (the widths as opposed to the shifts) of the relevant self-energy operators $\Sigma$ within $G$. These imaginary parts all have a common sign dictated by the imaginary part of the frequency $[\Sigma(\omega \pm i \varepsilon)=\Lambda(\omega)$ $\mp i \Gamma(\omega)]$ [2]. Hence Eq. (3) itself dictates the signs of the imaginary terms associated with any self-energy appearing in a denominator in terms of the relevant spectral frequency. Note, for example, that both longitudinal (population) and transverse (coherence) damping contribute to the damping factors, if with characteristic differences (for an examination of these in the Green's-function formalism see, for example, Ref. [20]). The damping factors obtained by combining the effects of all such processes must nonetheless themselves be signed in a consistent manner which is justified in this paper.

\section{SYMMETRY OF HARMONIC-GENERATION AMPLITUDES}

We now compare the symmetries of rival amplitudes under Hermitian conjugation and time reversal; for illustrative purposes we consider the coherent process of secondharmonic generation. The associated optical susceptibility tensor, obtained using the methods of Butcher and Cotter [9], has the form

$$
\begin{aligned}
\chi_{\alpha \beta \gamma}^{(2)}( & -2 \omega ; \omega, \omega) \\
= & \sum_{g} \sum_{b, c} \frac{\left\langle g\left|\mu_{\alpha}\right| c\right\rangle\left\langle c\left|\mu_{\beta}\right| b\right\rangle\left\langle b\left|\mu_{\gamma}\right| g\right\rangle}{\left(E_{g}-E_{b}+\hbar \omega+i \Gamma_{b}\right)\left(E_{g}-E_{c}+2 \hbar \omega+i \Gamma_{c}\right)} \\
& +\frac{\left\langle g\left|\mu_{\beta}\right| c\right\rangle\left\langle c\left|\mu_{\alpha}\right| b\right\rangle\left\langle b\left|\mu_{\gamma}\right| g\right\rangle}{\left(E_{g}-E_{b}+\hbar \omega+i \Gamma_{b}\right)\left(E_{g}-E_{c}-\hbar \omega-i \Gamma_{c}\right)} \\
& +\frac{\left\langle g\left|\mu_{\gamma}\right| c\right\rangle\left\langle c\left|\mu_{\beta}\right| b\right\rangle\left\langle b\left|\mu_{\alpha}\right| g\right\rangle}{\left(E_{g}-E_{b}-2 \hbar \omega-i \Gamma_{b}\right)\left(E_{g}-E_{c}-\hbar \omega-i \Gamma_{c}\right)} .
\end{aligned}
$$

The spectral theorem of quantum field theory (Sec. II) tells us to expect that the second-harmonic amplitude $\mathcal{A}_{\alpha \beta \gamma}^{(2)}(-2 \omega ; \omega, \omega)$ has the form

$$
\begin{aligned}
\mathcal{A}_{\alpha \beta \gamma}^{(2)}( & -2 \omega ; \omega, \omega) \\
= & \sum_{g} \sum_{b, c} \frac{\left\langle g\left|\mu_{\alpha}\right| c\right\rangle\left\langle c\left|\mu_{\beta}\right| b\right\rangle\left\langle b\left|\mu_{\gamma}\right| g\right\rangle}{\left(E_{g}-E_{b}+\hbar \omega+i \Gamma_{b}\right)\left(E_{g}-E_{c}+2 \hbar \omega+i \Gamma_{c}\right)} \\
& +\frac{\left\langle g\left|\mu_{\beta}\right| c\right\rangle\left\langle c\left|\mu_{\alpha}\right| b\right\rangle\left\langle b\left|\mu_{\gamma}\right| g\right\rangle}{\left(E_{g}-E_{b}+\hbar \omega+i \Gamma_{b}\right)\left(E_{g}-E_{c}-\hbar \omega+i \Gamma_{c}\right)} \\
& +\frac{\left\langle g\left|\mu_{\gamma}\right| c\right\rangle\left\langle c\left|\mu_{\beta}\right| b\right\rangle\left\langle b\left|\mu_{\alpha}\right| g\right\rangle}{\left(E_{g}-E_{b}-2 \hbar \omega+i \Gamma_{b}\right)\left(E_{g}-E_{c}-\hbar \omega+i \Gamma_{c}\right)}
\end{aligned}
$$


(such an expression has been employed in Ref. [21]). Equations (4) and (5) have identical structures, except that the imaginary terms in the denominators of the former have time-ordered signs while those of the latter have fixed signs.

When Hermitian conjugation $H$ is applied (for example, $\left.\langle c|O| b\rangle=\left\langle b\left|O^{\dagger}\right| c\right\rangle^{*}[9]\right), \chi_{\alpha \beta \gamma}^{(2)}$, but not $\mathcal{A}_{\alpha \beta \gamma}^{(2)}$, satisfies

$$
\left\{\chi_{\alpha \beta \gamma}^{(2)}(-2 \omega ; \omega, \omega)\right\}^{*}=\chi_{\alpha \beta \gamma}^{(2)}(2 \omega ;-\omega,-\omega) .
$$

In this standard semiclassical theory the susceptibility for any process must satisfy this type of relation in order for the Fourier transform and so the polarization to be a real quantity [9]. However, Berger [22] argues that the reciprocity relation of Eq. (6) (Berger's "microscopic inversibility") need not be a general symmetry.

Now consider the application of $H T$ symmetry, the combination of $H$ with time reversal $T$. We have, for example, that $\langle c|O| b\rangle=\left\langle\bar{b}\left|\bar{O}^{\dagger}\right| \bar{c}\right\rangle$, where $|\bar{b}\rangle=T|b\rangle$ and $\bar{O}=T O T^{-1}$ (see, for example, Ref. [23]). We assume that the unperturbed eigenstates are not split by a time-odd field, e.g., no Zeeman splittings, so that $|\bar{c}\rangle$ can be replaced by $|c\rangle$ in the sums and averages of the golden rule expression without affecting statistical factors or the denominators. It is also necessary to establish that the imaginary parts of the selfenergy $\Gamma_{b}$ are time even $\left(\Gamma_{b}=\Gamma_{\bar{b}}\right)$; this may be proved as follows. A many-body or resolvent formalism [1,2] leads to the Brillouin-Wigner perturbative expansion of the selfenergy in the interactions $V$ :

$$
\Sigma_{b}(E+i \varepsilon)=\sum_{n=0}^{\infty}\left\langle b\left|V\left(\frac{Q}{E+i \varepsilon-H_{0}} V\right)^{n}\right| b\right\rangle
$$

here $H_{0}$ is the unperturbed Hamiltonian and $Q$ a projection operator off the level $b, E \rightarrow E_{b}, \varepsilon \rightarrow 0^{+}$. Each denominator in this expression may resonate, giving an imaginary part $\Gamma_{b}(E)$, which, using the limit $1 /(x+i \varepsilon) \rightarrow P(1 / x)-i \pi \delta(x)$, takes the form

$$
\begin{aligned}
\Gamma_{b}(E)= & \pi \sum_{l, m=0}^{\infty} \sum_{c \neq b}\left\langle b\left|V\left(\frac{Q}{E-H_{0}} V\right)^{l}\right| c\right\rangle \\
& \times\left\langle c\left|V\left(\frac{Q}{E-H_{0}} V\right)^{m}\right| b\right\rangle \delta\left(E-E_{c}\right) \\
= & \pi \sum_{l, m=0}^{\infty} \sum_{c \neq b} P_{b c}^{(l)} P_{c b}^{(m)} \delta\left(E-E_{c}\right),
\end{aligned}
$$

where, writing $V_{b c}$ for $\langle b|V| c\rangle$, we have

$$
P_{b c}^{(l)}=\sum_{d, e, \cdots, g} \frac{\overbrace{V_{b d} V_{d e} \cdots V_{g c}}^{l}}{\left(E-E_{d}\right) \cdots\left(E-E_{g}\right)}=P_{c b}^{(l) *}=P_{\bar{c} \bar{b}}^{(l)} .
$$

The last symmetries, which derive from Hermiticity and time-reversal invariance when states $d, \bar{d}$, etc., are degenerate, together with the palindromic structure of Eq. (8), show that $\Gamma_{b}(E)=\Gamma_{\bar{b}}(E)$. Therefore, the amplitude $\mathcal{A}_{\alpha \beta \gamma}^{(2)}$ with fixed signs, but not $\chi_{\alpha \beta \gamma}^{(2)}$ with the time-ordered signs, is seen to satisfy

$$
\mathcal{A}_{\alpha \beta \gamma}^{(2)}(-2 \omega ; \omega, \omega)=\mathcal{A}_{\alpha \beta \gamma}^{(2)}(2 \omega ;-\omega,-\omega) .
$$

Equation (10) proves the formal identity required of the second-harmonic generation and parametric downconversion amplitudes. It is a general consequence of the applicability of time-reversal symmetry. Formally, the evolution operator for a closed light-matter system has the solution $\exp (-i / \hbar \mathcal{H} t)$, where $\mathcal{H}$ is the total Hamiltonian describing all relevant degrees of freedom such as those associated with the rotational, vibrational, and translational modes of particles making up the system. Supposing that the initial and final states of the system are $I, F$, respectively, $H T$ symmetry gives

$$
\left\langle F\left|e^{-i / \hbar \mathcal{H} t}\right| I\right\rangle=\left\langle\bar{I}\left|e^{-i / \hbar \mathcal{H} t}\right| \bar{F}\right\rangle
$$

[see the work of Stedman [3], whose "reversal theorem" is related to Eq. (11)]. Assuming that the internal degrees of freedom of the system are initially at equilibrium and that the system is not magnetic, Eq. (11) proves the equivalence of time-reversed processes without recourse to explicit perturbative expansions. We have seen for second-harmonic generation that as part of this equivalence all the imaginary addenda must have the same sign. It follows similarly that this principle must also hold for higher-order transition amplitudes. Note that the time-reversal symmetry relation of Eq. (11) would not be expected to hold if the system were coupled to a bath because that is no longer a thermodynamically isolated system. However, the generality of $\mathcal{H}$ means that the various damping mechanisms need not be represented by couplings to external influences. Further analyses of the ensemble "arrow of time" do not impinge upon our discussion since they do not address the physics of time reversal at the fundamental level of photon interaction [24].

The second-harmonic intensity expressions are given by $\left|\chi^{(2)}\right|^{2}$ and $\left|\mathcal{A}^{(2)}\right|^{2}$ in the time-ordered-sign and fixed-sign formulations, respectively. When damping is included Eqs. (4) and (5) show that $\chi^{(2)}$ and $\mathcal{A}^{(2)}$ are not proportional, nor do they stand in a complex conjugate relationship. Therefore, those alternative formulations lead to intensity expressions that in principle are physically distinguishable. The denominator factors of $\chi^{(2)}$ that may be resonant have the correct positive imaginary parts; only the "antiresonant" factors have negative imaginary parts. Hence the difference between results cast in terms of $\chi^{(2)}$ and $\mathcal{A}^{(2)}$ is generally likely to be small in applications. Nevertheless, the difference is in principle measurable and in certain cases quite significant (see below). At this level of discussion the importance of these corrections might be judged to be similar to that of departures from the rotating-wave approximation, which itself depends on retaining only resonant terms.

\section{QUALITATIVE CONSEQUENCES}

We now illustrate how the two sign conventions not only give inequivalent quantitative predictions but also give differing qualitative predictions. To do this we consider the 
coherent process of electro-optic rotation (forward Rayleigh scattering between orthogonal linear polarizations in the presence of an external electric field) in fluid media. The associated expressions in the time-ordered and fixed-sign conventions are, respectively,

$$
\begin{aligned}
& \chi_{\alpha \beta \gamma}^{(2)}(-\omega ; 0, \omega)=\sum_{g} \sum_{b, c} \frac{\mu_{g c}^{\alpha} \mu_{c b}^{\beta} \mu_{b g}^{\gamma}}{\left(E_{g b}+i \Gamma_{b}\right)\left(E_{g c}+\hbar \omega+i \Gamma_{c}\right)} \\
& +\frac{\mu_{g c}^{\gamma} \mu_{c b}^{\alpha} \mu_{b g}^{\beta}}{\left(E_{g b}+\hbar \omega+i \Gamma_{b}\right)\left(E_{g c}-i \Gamma_{c}\right)} \\
& +\frac{\mu_{g c}^{\beta} \mu_{c b}^{\gamma} \mu_{b g}^{\alpha}}{\left(E_{g b}-\hbar \omega-i \Gamma_{b}\right)\left(E_{g c}-\hbar \omega-i \Gamma_{c}\right)} \\
& +\frac{\mu_{g c}^{\alpha} \mu_{c b}^{\gamma} \mu_{b g}^{\beta}}{\left(E_{g b}+\hbar \omega+i \Gamma_{b}\right)\left(E_{g c}+\hbar \omega+i \Gamma_{c}\right)} \\
& +\frac{\mu_{g c}^{\beta} \mu_{c b}^{\alpha} \mu_{b g}^{\gamma}}{\left(E_{g b}+i \Gamma_{b}\right)\left(E_{g c}-\hbar \omega-i \Gamma_{c}\right)} \\
& +\frac{\mu_{g c}^{\gamma} \mu_{c b}^{\beta} \mu_{b g}^{\alpha}}{\left(E_{g b}-\hbar \omega-i \Gamma_{b}\right)\left(E_{g c}-i \Gamma_{c}\right)}, \\
& \mathcal{A}_{\alpha \beta \gamma}^{(2)}(-\omega ; 0, \omega)=\sum_{g} \sum_{b, c} \frac{\mu_{g c}^{\alpha} \mu_{c b}^{\beta} \mu_{b g}^{\gamma}}{\left(E_{g b}+i \Gamma_{b}\right)\left(E_{g c}+\hbar \omega+i \Gamma_{c}\right)} \\
& +\frac{\mu_{g c}^{\gamma} \mu_{c b}^{\alpha} \mu_{b g}^{\beta}}{\left(E_{g b}+\hbar \omega+i \Gamma_{b}\right)\left(E_{g c}+i \Gamma_{c}\right)} \\
& +\frac{\mu_{g c}^{\beta} \mu_{c b}^{\gamma} \mu_{b g}^{\alpha}}{\left(E_{g b}-\hbar \omega+i \Gamma_{b}\right)\left(E_{g c}-\hbar \omega+i \Gamma_{c}\right)} \\
& +\frac{\mu_{g c}^{\alpha} \mu_{c b}^{\gamma} \mu_{b g}^{\beta}}{\left(E_{g b}+\hbar \omega+i \Gamma_{b}\right)\left(E_{g c}+\hbar \omega+i \Gamma_{c}\right)} \\
& +\frac{\mu_{g c}^{\beta} \mu_{c b}^{\alpha} \mu_{b g}^{\gamma}}{\left(E_{g b}+i \Gamma_{b}\right)\left(E_{g c}-\hbar \omega+i \Gamma_{c}\right)} \\
& +\frac{\mu_{g c}^{\gamma} \mu_{c b}^{\beta} \mu_{b g}^{\alpha}}{\left(E_{g b}-\hbar \omega+i \Gamma_{b}\right)\left(E_{g c}+i \Gamma_{c}\right)},
\end{aligned}
$$

where $E_{g b}=E_{g}-E_{b}$. The application of $H T$ shows that the amplitude with fixed signs, but not that with time-ordered signs, satisfies

$$
\mathcal{A}_{\alpha \beta \gamma}^{(2)}(-\omega ; 0, \omega)=\mathcal{A}_{\beta \alpha \gamma}^{(2)}(-\omega ; 0, \omega) .
$$

In a fluid we detect only the rotationally invariant part and must contract these tensors with the Levi-Cività symbol $\epsilon_{\alpha \beta \gamma}$ (see, for example, Ref. [25]). However, such an antisymmetric combination is incompatible with the index symmetry of Eq. (14). Hence, in the correct formulation this process is forbidden, whereas it is allowed under the popular alternative sign choice. Such qualitative differences in prediction are particularly amenable to experimental test.

Manakov and Fănshteı̆n [26] and Agre and Manakov [27] also discuss the role of damping factors in stimulating various nonlinear optical processes (through the interference of the anti-Hermitian and Hermitian terms in the transition amplitude). These authors assume a time-odd character for the damping constant in contradiction to our result. It would appear from Manakov and Fainshtein [26] that they reach this erroneous conclusion because they have assumed the timereversal covariance of a diffusion equation of the form $d W / d t=-\gamma W$. However, thermodynamic diffusion effects reflect the arrow of time and we prove that the width parameter $\Gamma$ is demonstrably time even. This will have significant consequences for their analysis [26,27].

\section{SOME OTHER LIMITATIONS OF SEMICLASSICAL APPROACHES}

Why should the time-ordered-sign formalism be misleading and inaccurate, given that it reflects causality considerations in the theory of the semiclassical susceptibility? The amplitudes with fixed signs are based on resolvent or field theoretic techniques and directly calculate the observables of quantum theory. The susceptibility formulation has a more semiclassical foundation. The description of optical response in terms of susceptibility, whether linear or nonlinear, is not universally appropriate. It derives from a tradition long established in classical optics, wherein a material polarization is regarded as the source of any emergent signal. This source, and hence a forteriori the various orders of susceptibility in terms of which it is cast through series expansion, has the status of an inferred rather than an experimentally determinable quantity. In the case of laser optics, at least, where nonlinearities are usually engendered by pulsed radiation and where genuinely photonic aspects of the radiation can be of paramount importance, such an assumption is a gross oversimplification. For example, it leads to the obviously false conclusion that any system exposed to even one photon can, through quadratic interaction mediated by a second-order susceptibility, weakly generate second-harmonic output.

It should not be assumed that the issues we have addressed are simply reflections of a difference originating from a Fock state quantum electrodynamical basis. If quasiclassical coherent states are employed for the radiation, damping materializes in exactly the same fashion as we have described and its sign is correctly determined by precisely the same considerations. However, there are other reasons to doubt the utility of the semiclassical susceptibility formulation, for example, the obscurity it casts upon certain kinds of symmetry analysis. This is forcibly illustrated by controversies over the rigorous preclusion in fluid media of secondharmonic generation, to all orders of multipolar approximation [28]. Here too one can trace problems arising from necessarily identifying signals with a dipolar source, another feature at the conceptual heart of the susceptibility formalism. We believe that the proper formulation of any optical process requires its expression in terms of direct observables, principally rates and signal intensities, rather than the (at best) inferred optical susceptibilities.

\section{CONCLUSIONS}

In the case of weak resonances the simplest expedient for dealing with singular energy denominators is to incorporate within each denominator factor a small imaginary addendum, expressly for the purpose of obtaining the desired analytic properties. Time-reversal symmetry requires all those 
addenda to have the same sign. This conclusion is consistent with fully quantum-mechanical derivations. The damping signs usually associated with the semiclassical optical susceptibility methods are in conflict with this symmetry and the intensity expressions that follow differ from the correct expression both quantitatively and qualitatively.

\section{ACKNOWLEDGMENTS}

D.L.A. is grateful to the University of Canterbury for financial support during the tenure of which this work was begun. S.N. is grateful to the University of Canterbury for financial support.
[1] C. Cohen-Tannoudji, J. Dupont-Roc, and G. Grynberg, AtomPhoton Interactions (Wiley-Interscience, New York, 1992), pp. 222-238.

[2] G. E. Stedman, Am. J. Phys. 39, 205 (1971).

[3] G. E. Stedman, Diagram Techniques in Group Theory (Cambridge University Press, Cambridge, 1990), pp. 174-217.

[4] B. J. McKenzie and G. E. Stedman, J. Phys. C 11, 589 (1978).

[5] D. P. Craig and T. Thirunamachandran, Molecular Quantum Electrodynamics (Academic, London, 1984).

[6] L. Allen and J. H. Eberly, Optical Resonance and Two-Level Atoms (Wiley-Interscience, New York, 1975).

[7] V. S. Butylkin, A. E. Kaplan, Yu. G. Khronopulo, and E. I. Yakubovich, Resonant Nonlinear Interactions of Light with Matter (Springer-Verlag, Berlin, 1989).

[8] G. Juzeliūnas and D. L. Andrews, Phys. Rev. B 49, 8751 (1994); 50, 13371 (1994).

[9] P. N. Butcher and D. Cotter, The Elements of Nonlinear Optics (Cambridge University Press, Cambridge, 1990), pp. 92, 93, and 132 .

[10] N. Bloembergen, Nonlinear Optics (Benjamin, New York, 1965), p. 26.

[11] C. Flytzanis, Quantum Electronics Vol. 1 Pt. A, edited by H. Rabin and C. L. Tang (Academic, New York, 1975), pp. 9207.

[12] Y. R. Shen, The Principles of Nonlinear Optics (WileyInterscience, New York, 1984), pp. 14-17 and 86.

[13] M. D. Levenson and S. S. Kano, Introduction to Nonlinear Laser Spectroscopy (Revised edition) (Academic, Boston, 1988), pp. 63 and 66.
[14] P. N. Butcher, R. Loudon, and T. P. McLean, Proc. Phys. Soc. (GB) 85, 565 (1965).

[15] D. C. Hanna, M. A. Yuratich, and D. Cotter, in Nonlinear Optics of Free Atoms and Molecules, edited by D. L. MacAdam, Springer Series in Optical Sciences Vol. 17 (SpringerVerlag, Berlin, 1979), p. 34.

[16] L. Hecht and L. D. Barron, Mol. Phys. 79, 887 (1993).

[17] V. Weisskopf, Ann. Phys. (Leipzig) 9, 23 (1931); V. Weisskopf, Z. Phys. 85, 451 (1933).

[18] T. D. Schultz, Quantum Field Theory and the Many-Body Problem (Gordon and Breach, London, 1964).

[19] R. Barrie and R. G. Rystephanick, Can. J. Phys. 44, 109 (1966).

[20] G. E. Stedman, J. Phys. C 4, 1022 (1971).

[21] T. L. Mazely and W. M. Hetherington, J. Chem. Phys. 86, 3640 (1987).

[22] J. Berger, Eur. J. Phys. 11, 155 (1990).

[23] S. Naguleswaran and G. E. Stedman, J. Phys. B 29, 4027 (1996).

[24] R. G. Sachs, The Physics of Time Reversal (University of Chicago Press, Chicago, 1987), p. 30.

[25] D. L. Andrews and T. Thirunamachandran, J. Chem. Phys. 67, 5026 (1977).

[26] N. L. Manakov and A. G. Faunshtěn, Zh. Éksp. Teor. Fiz. 87, 1552 (1984) [Sov. Phys. JETP 60, 890 (1984)].

[27] M. Ya Agre and N. L. Manakov, J. Phys. B 29, L7 (1996).

[28] D. L. Andrews, Modern Nonlinear Optics, Advances in Chemical Physics Vol. LXXXV, edited by M. Evans and S. Kielich (Wiley, New York, 1993), pp. 545-606. 